\title{
Mathematical Model for the Control of measles
}

\section{1*PETER, OJ; ${ }^{2}$ AFOLABI, OA; ${ }^{3}$ VICTOR, AA; ${ }^{4}$ AKPAN, CE; ${ }^{5}$ OGUNTOLU, FA}

\author{
${ }^{1,2,3}$ Department of Mathematics, University of Ilorin, Ilorin, Kwara State, Nigeria, \\ ${ }^{4}$ Departmennt of Computer Science, Obong University, Obong Ntak. Akwa Ibom State, Nigeria \\ ${ }^{5}$ Department of Mathematics/Statistics, Federal University of Technology, Minna, Nigeria \\ *Corresponding author Email: peterjames4real@gmail.com, +2348033560280
}

\begin{abstract}
We proposed a mathematical model of measles disease dynamics with vaccination by considering the total number of recovered individuals either from natural recovery or recovery due to vaccination. We tested for the existence and uniqueness of solution for the model using the Lipchitz condition to ascertain the efficacy of the model and proceeded to determine both the disease free equilibrium (DFE) and the endemic equilibrium (EE) for the system of the equations and vaccination reproduction number are given. Numerical simulation of the model shows that vaccination is capable of reducing the number of exposed and infectious population.
\end{abstract}

\section{DOI: https://dx.doi.org/10.4314/jasem.v22i4.24}

Copyright: Copyright $\left({ }^{\circ} 2018\right.$ Peter et al. This is an open access article distributed under the Creative Commons Attribution License (CCL), which permits unrestricted use, distribution, and reproduction in any medium, provided the original work is properly cited.

Dates: Received: 13 March; Revised: 16 April: 2018; Accepted: 22 April 2018

Keywords: Measles, Vaccination, Equilibrium States, Basic Reproduction Number.

Measles is an infection of the respiratory system caused by a virus of the genus Morbillivirus. The disease is spread through respiration following contact with fluids from an infected person's nose and mouth, either directly or indirectly. The disease is highly contagious with $90 \%$ chances of being infected by individuals (without immunity). Measles infects about 30 to 40 million children every year. The disease has been, and remains, a major killer of children around the world. Despite the introduction of the measles vaccine in 1963, measles caused an estimated 2.6 million deaths in a single year as far back as 1980 . According to WHO, in 2002 alone, the disease is estimated to have caused 614000 death globally, with more than half of fatalities occur in sub-saharan Africa. Survivor children subsequently suffer blindness, deafness or impaired vision, brain damage, and death (WHO, 2005). The control of measles is largely based on the use of MMR (measles, mumps, and rubella) and MMRV (measles, mumps, rubella, and varicella) vaccines, which are believed to be about 95\% effective. Worldwide, measles vaccination has been very effective, preventing an estimated 80 million cases and 4.5 million deaths annually (Simons et al, 2012). Although global incidence has been significantly reduced through vaccination, measles remains an important public health problem. Since vaccination coverage is not uniformly high worldwide, measles stands as the leading vaccine-preventable killer of children worldwide; measles is estimated to have caused 614000 global deaths annually in 2002, with more than half of measles deaths occur in subsaharan Africa (Grais et al., 2006)... (Ejima, et al 2012). The following are some of the mathematical models developed to study transmission dynamics of measles disease using different approaches: (Bakare et al., 2012), Studied modeling and simulation of the dynamics of the transmission of measles disease. They used SEIR model to discuss dynamics of measles infection and address the stability and disease free and endemic equilibrium states. The impact of vaccination in the control and elimination of measles was not considered in work.

(Bolarin, 2014) Based his study on the dynamical analysis of a new model for measles infection. His study used SEIR model modified by adding vaccinated compartment. His model determined the required vaccination coverage and dosage that will guarantee eradication of measles disease within a population. (Verguet, 2015). Performed a study on controlling measles using supplemental immunization activities: a mathematical model to inform optimal policy. They developed DynaMICE (Dynamic Measles Immunization Calculation Engine), an age-stratified model of measles infection transmission in vaccinated and unvaccinated individuals. The population in the model can be susceptible to measles, infected with measles or recovered from measles (and hence have lifelong immunity) that is SIR model. In their study, the rate at which infection occurs in the susceptible population depends on the existing proportion of the 
population that is already infected, as well as the effective contact rate between different age groups. Individuals age discretely, in one-year increments, at the end of each year between 0 and 100 years old. Furthermore, both numerical simulation and mathematical analysis indicated that a single Supplemental Immunization Activity (SIA) will not control measles disease outbreaks in any of the countries with high burden of measles. However, regular Supplemental Immunization Activities (SIAs) at high coverage are able to control measles transmission, with the periodicity of SIA campaigns determined by population demo-graphics and existing MCV1 coverage. (Momoh, 2015). Developed a mathematical model for control of measles epidemiology. They used SEIR model to determine the impact of exposed individuals at latent period through the stability analysis and numerical simulation. (Catherine, 1998). Developed a mathematical model for measles epidemics in Ireland. The aim of her study was to establish a mathematical model for measles epidemics and to predict the levels of vaccination coverage required in Ireland in order to eradicate the disease. The aim of this paper is to show that in the presence of vaccination, a modified threshold parameter which is called the vaccine reproduction number will have the value less than one for a certain level of vaccination

\section{MATERIALS AND METHODS}

We formulate a non-linear deterministic model for the transmission dynamics of infectious diseases .The model subdivides the human population into five (5) compartments depending on the epidemiological status of individuals. The compartments are susceptible $S$, Vaccinated V Expose E, infected $I$ and the recovered $R$. We assume that MMR vaccine moves individuals from the susceptible compartment to the vaccinated compartment at a rate $\varphi$, and an individual into recovered compartment because immune due to vaccination at a rate $r$. We assumed a natural death rate in each class $\mu$. $>0$.To indicate this mathematically, we have.

$$
\left.\begin{array}{rl}
S^{\prime} & =\Lambda-\lambda S-(\mu+\varphi) S \\
V^{\prime} & =\varphi S-(1-\varphi) \lambda V-(\mu+r) V \\
E^{\prime} & =\alpha \lambda S+(1-\phi) \lambda V-\delta_{1} \lambda E-(\mu+\omega) E+\alpha_{1} \delta_{2} R \\
I^{\prime} & =(1-\alpha) \lambda S+\delta_{1} \lambda E+\omega E-(\mu+\rho+d) I+\left(1-\alpha_{1}\right) \delta_{2} \lambda R \\
R^{\prime} & =r V+\rho I-\mu R-\delta_{2} \lambda R
\end{array}\right\}
$$

Where $\lambda=\frac{\beta I}{N}$

The total population size is:

$N=S+V+E+I+R$

Existence and Uniqueness of Solution: The validity and authenticity of any mathematical model depends on whether the given system of equations has a solution, and if the solution is unique. We shall use the Lipchitz condition to verify the existence and uniqueness of solution for the system of equations 1

Let the system of equation 1 be as follows

$$
\begin{aligned}
& F_{1}=\Lambda-\lambda S-(\mu+\varphi) S \\
& F_{2}=\varphi S-(1-\varphi) \lambda V-(\mu+r) V \\
& F_{3}=\alpha \lambda S+(1-\phi) \lambda V-\delta_{1} \lambda E-(\mu+\omega) E+\alpha_{1} \delta_{2} R \\
& F_{4}=(1-\alpha) \lambda S+\delta_{1} \lambda E+\omega E-(\mu+\rho+d) I+\left(1-\alpha_{1}\right) \delta_{2} \lambda R \\
& F_{5}=r V+\rho I-\mu R-\delta_{2} \lambda R
\end{aligned}
$$

Theorem 1 (Derrick and Groosman,1976): Let D denote the region

$$
\begin{aligned}
& \left|t-t_{0}\right| \leq a,\left\|x-x_{0}\right\| \leq 1, x= \\
& \left(x_{1}, x_{2} \ldots \ldots, x_{n}\right), x_{0}=\left(x_{10}, x_{20}, \ldots \ldots \ldots x_{n o}\right)
\end{aligned}
$$

And suppose that $f(t, x)$ satisfies the Lipchitz condition

$\left\|f\left(t, x_{1}\right)-f\left(t, x_{2}\right)\right\| \leq k\left\|x_{1}-x_{2}\right\|$ whenever the pairs $\left(t, x_{1}\right)$ and $\left(t, x_{2}\right)$ belong to $D$ where $k$ is a positive constant. Then, there is a constant $\delta \geq 0$ such that there exists a unique continuous vector solution of $x(t)$ of the system in the interval $t-t_{o} \leq \delta$. It is important to note that the condition is satisfied by the requirement that $\frac{\partial f_{i}}{\partial x_{j}}, i, j=1,2, \cdots$, be continuous and bounded in $D$ Considering the model equations 2 , we are interested in the region $0 \leq \alpha \leq R$. We look for the bounded solution in the region and whose partial derivatives satisfy $f \leq \alpha \leq 0$. where $\alpha$ and $\delta$ are positive constants.

Theorem 2: Let $\mathrm{D}$ denote the region $0 \leq \alpha \leq R$, then equation 2 have a unique solution. We show that

$\frac{\partial f_{i}}{\partial x_{j}}, i, j=1,2,3,4,5$

Are continuous and bounded in D For $F_{1}$

$\left|\frac{\partial f_{1}}{\partial S}\right|=|-\lambda-(\mu+\varphi) S|<\infty$,

$\left|\frac{\partial f_{1}}{\partial V}\right|=0<\infty \quad\left|\frac{\partial f_{1}}{\partial E}\right|=0<\infty$

$\left|\frac{\partial f_{1}}{\partial I}\right|=0<\infty\left|\frac{\partial f_{1}}{\partial R}\right|=0<\infty$

For $F_{2}$ 


$$
\begin{aligned}
& \left|\frac{\partial f_{2}}{\partial S}\right|=|\varphi|<\infty \\
& \left|\frac{\partial f_{2}}{\partial V}\right|=|-(1-\phi) \lambda-(\mu+r)|<\infty \\
& \left|\frac{\partial f_{2}}{\partial E}\right|=0<\infty,\left|\frac{\partial f_{2}}{\partial I}\right|=0<\infty,\left|\frac{\partial f_{2}}{\partial R}\right|=0<\infty
\end{aligned}
$$

For $F_{3}$

$$
\begin{aligned}
& \left|\frac{\partial f_{3}}{\partial S}\right|=|\alpha \lambda \quad|<\infty,\left|\frac{\partial f_{3}}{\partial V}\right|=|(1-\phi) \lambda|<\infty \\
& \left|\frac{\partial f_{3}}{\partial E}\right|=\left|-\delta_{1} \lambda-(\mu+\omega)\right|<\infty\left|\frac{\partial f_{3}}{\partial I}\right|=0<\infty \text {, } \\
& \left|\frac{\partial f_{3}}{\partial R}\right|=\left|\alpha_{1} \delta_{2}\right|<\infty
\end{aligned}
$$

For $F_{4}$

$$
\begin{aligned}
& \left|\frac{\partial f_{4}}{\partial S}\right|=|1-\alpha| \lambda<\infty,\left|\frac{\partial f_{4}}{\partial V}\right|=0<\infty, \\
& \left|\frac{\partial f_{4}}{\partial E}\right|=\left|\delta_{1} \lambda+\omega\right|<\infty \\
& \left|\frac{\partial f_{4}}{\partial I}\right|=|-(\mu+p+d)|<\infty \\
& \left|\frac{\partial f_{4}}{\partial R}\right|=\left|1-\alpha_{1}\right| \delta_{2} \lambda<\infty
\end{aligned}
$$

For $F_{5}$

$$
\begin{aligned}
& \left|\frac{\partial f_{5}}{\partial S}\right|=0<\infty\left|\frac{\partial f_{5}}{\partial V}\right|=|r|<\infty \\
& \left|\frac{\partial f_{5}}{\partial E}\right|=0<\infty
\end{aligned}
$$

$$
=\left(\frac{\Lambda}{\mu+\varphi+\lambda}, \frac{\Lambda \varphi}{(\mu+\varphi+\lambda)(\mu+r+[1-\phi] \lambda)}, E^{*}, I^{*}, R^{*}\right)
$$

\section{Where}

$$
\begin{aligned}
E^{*} & =\frac{\alpha \lambda \Lambda(\mu+r+[1-\phi] \lambda)\left(\mu+\delta_{2} \lambda\right)+[1-\phi] \lambda \varphi\left(\mu+\delta_{2} \lambda\right)+\alpha \delta_{2} \lambda\left(r \varphi \Lambda+p(\mu+\varphi+\lambda)(\mu+r[1-\phi] \lambda) I_{1}\right.}{\left(\mu+\omega+\delta_{1} \lambda\right)\left(\mu+\delta_{2} \lambda\right)(\mu+\varphi+\lambda)(\mu+r+[1-\phi] \lambda)} \\
I^{*} & =\frac{(1-\alpha) \lambda S+\left(\delta_{1} \lambda+\omega\right) E+\left(1-\alpha_{1}\right) \delta_{2} \lambda R}{\mu+p+d} \\
R^{*} & =\frac{r \varphi \Lambda+\rho(\mu+\varphi+\lambda)+(\mu+r[1-\phi] \lambda) I}{\left(\mu+\delta_{2} \lambda\right)(\mu+\varphi+\lambda)(\mu+r+[1-\phi] \lambda)}
\end{aligned}
$$

The basic reproduction number of vaccination by using Next generation matrix approch (stephen et al, 2014) is given by

$$
R_{v}=\frac{\beta \Lambda[\mu(\mu+r)(1-\alpha) \mu+\omega]+\left[\left(1-\alpha_{1}\right) \mu+\omega\right] \delta_{2} \varphi r+(1-\phi) \omega \varphi \mu}{\mu N(\mu+r)(\mu+\omega)(\mu+d+\rho)}
$$

Proposition 1: The disease free equilibrium is locally asymtotically stable when the vaccination reproduction number $\mathrm{R}_{\mathrm{v}}<1$ and unstable if $\mathrm{R}_{\mathrm{v}}>1$

Proof

Let 


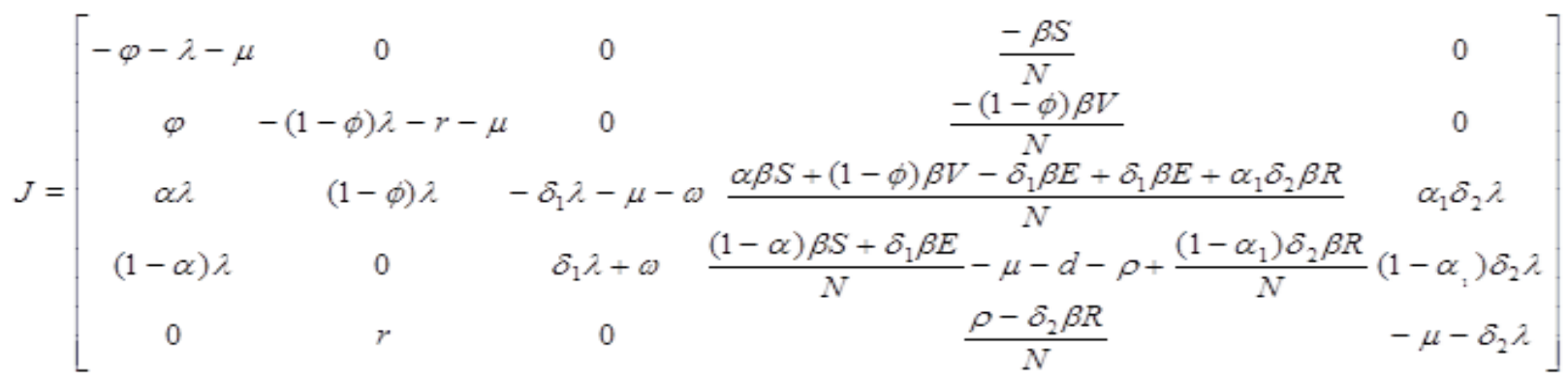

The Jacobian matrix at $(D F E) E_{0}$ is given as

$$
J\left(E_{0}\right)=\left[\begin{array}{ccccc}
-\varphi-\mu & 0 & 0 & \frac{-\beta \Lambda}{(\mu+\varphi) \beta V} & 0 \\
\varphi & -r-\mu & 0 & \frac{-(1-\phi) \beta \varphi \Lambda}{(\mu+r)(\mu+\varphi) N} & 0 \\
0 & 0 & -\mu+\omega & \frac{\alpha \beta \Lambda}{(\mu+\varphi) N}+\frac{(1-\phi) \beta \varphi \Lambda}{(\mu+r)(\mu+\varphi) N}+\frac{\alpha_{1} \delta_{2} \beta \varphi r \Lambda}{\mu(\mu+r)(\mu+\varphi) N} & 0 \\
0 & 0 & \omega & \frac{(1-\alpha) \beta S+\delta_{1} \beta E}{N}-\mu-d-\rho+\frac{\left(1-\alpha_{1}\right) \delta_{2} \beta R}{N} & 0 \\
0 & r & 0 & \rho-\frac{\delta_{2} \beta \varphi \Lambda}{\mu(\mu+r)(\mu+\varphi) N} & -\mu
\end{array}\right]
$$

$\operatorname{De}\left\{J\left(E_{0}\right)\right]=\beta \Lambda[\mu(\mu+r)(1-\alpha) \mu+\omega]+\left[\left(1-\alpha_{1}\right) \mu+\omega\right] \delta_{2} \varphi r+(1-\phi) \omega \varphi \mu-\mu N(\mu+r)(\mu+\omega)(\mu+\varphi)(\mu+d+\rho)$ $\operatorname{Det} J\left(E_{0}\right)>0$ when $\frac{\beta \Lambda[\mu(\mu+r)(1-\alpha) \mu+\omega]+\left[\left(1-\alpha_{1}\right) \mu+\omega\right] \delta_{2} \varphi r+(1-\phi) \omega \varphi \mu}{\mu N(\mu+r)(\mu+\omega)(\mu+\varphi)(\mu+d+\rho)}=R_{v}<1$

The real part of the eigen values of the real part of the eigen value of $\operatorname{Det}\left[J\left(E_{0}\right)-\lambda\right]=0$ $\operatorname{Det}\left[J\left(E_{0}\right)-\lambda\right]=0$ are all negative when $R_{v}<1$. (Sarah, 2012) This shows that $E_{0}$ is locally asymptotically stable whenever $R_{v}<1$ On the contrary, $R_{0}=\frac{\beta \Lambda(1-\alpha)(\mu+\omega)}{\mu N(\mu+\omega)(\mu+d+\rho)}$

As a result,

$R_{v}=R_{0}\left(\frac{\beta \Lambda[\mu(\mu+r)(1-\alpha) \mu+\omega]+\left[\left(1-\alpha_{1}\right) \mu+\omega\right] \delta_{2} \varphi r+(1-\phi) \omega \varphi \mu}{\mu N(\mu+r)(\mu+\omega)(\mu+\varphi)(\mu+d+\rho)}\right)<R_{0}$
$R_{v}=R_{0}\left(\frac{(\mu+r)\left([1-\alpha] \mu^{2}+\omega \mu\right]+\left[\left(1-\alpha_{1}\right) \mu+\omega\right] \delta_{2} \varphi r+(1-\phi) \omega \varphi \mu}{(\mu+r)(\mu+\varphi)[(1-\alpha) \mu+\omega]}\right)<R_{0}$

Since

$(\mu+r)\left([1-\alpha] \mu^{2}+\omega \mu\right]+\left[\left(1-\alpha_{1}\right) \mu+\omega\right] \delta_{2} \varphi r+(1-\phi) \omega \varphi \mu<(\mu+r)(\mu+\varphi)[(1-\alpha) \mu+\omega]$

Hence,for $(\varphi>0$ and $0<\phi \leq 1)$ the effect of vaccine will always reduce the effect of the basic reptoduction number $R_{0}$ from the disease.

Furthermore, let $\mathrm{R}_{\mathrm{v}}=1$ and solve for $\varphi$ so that the condition is $\varphi^{c}$. The solution is a vaccination critical level given by:

$$
\begin{aligned}
& \frac{\beta \Lambda[\mu(\mu+r)(1-\alpha) \mu+\omega]+\left[\left(1-\alpha_{1}\right) \mu+\omega\right] \delta_{2} \varphi r+(1-\phi) \omega \varphi \mu}{\mu N(\mu+r)(\mu+\omega)(\mu+\varphi)(\mu+d+\rho)}=1 \\
& \beta \Lambda[\mu(\mu+r)(1-\alpha) \mu+\omega]+\left[\left(1-\alpha_{1}\right) \mu+\omega\right] \delta_{2} \varphi r+(1-\phi) \omega \varphi \mu=\mu N(\mu+r)(\mu+\omega)(\mu+\varphi)(\mu+d+\rho) \\
& \varphi^{c}=\frac{\mu^{2} N(\mu+r)(\mu+\omega)(\mu+d+\rho)-\beta \Lambda(\mu(\mu+r)[(1-\alpha) \mu+\omega])}{\left.\beta \Lambda\left(\left[1-\alpha_{1}\right) \mu+\omega\right] \delta_{2} \varphi r+(1-\phi) \omega \mu\right)-\mu N(\mu+r)(\mu+\omega)(\mu+d+\rho)}
\end{aligned}
$$




$$
=\frac{\left.\mu\left(1-R_{0}\right)(\mu+r)[(1-\alpha) \mu+\omega]\right)}{\left.\left(\left[1-\alpha_{1}\right) \mu+\omega\right] \delta_{2} \varphi r+(1-\phi) \omega \mu\right)} R_{0}-1
$$

We can conclude that if $\varphi<\varphi^{c}$ vaccination will not be effective in eradicating the disease, also if $\varphi>\varphi^{c}$ vaccination will be effective and this will eliminate the endemic condition

\section{RESULTS AND DISCUSSION}

Numerical simulations of the model were carried out to graphically illustrate the trend of exposed, infected, and recovered populations in Figure 1, Figure 2 and Figure 3 below. The Table below shows the set of parameter values and the state variables which were used in order to support the analytical results,

Table 1: Description of Parameters and vslues for the Model

\begin{tabular}{|c|c|c|c|}
\hline Parameters & Interpretation & Values & Source \\
\hline$\Lambda$ & recruitment rate into susceptible class & 200 & Assumed \\
\hline$\varphi$ & vaccination rate from $S$ & 0.7 & (Stephen et al, 2014 \\
\hline$\mu$ & Natural death rate & 0.00875 per year & $\begin{array}{l}\text { (Stephen et al, } \\
\text { 2014) }\end{array}$ \\
\hline$\phi$ & vaccination effectiveness & 0.02 & Assumed \\
\hline$d$ & natural death rate for I & 0.125 & (Freed et al,2014) \\
\hline$\beta$ & transmission probabilities & 0.09091 per year & (Anes,2012). \\
\hline$\omega$ & Natural rate of progression to I & 0.0013 & Assumed \\
\hline$\alpha_{1}$ & probability of $R$ entering into $E$ & 0.14 & Assumed \\
\hline$\alpha$ & probability of $S$ entering into $E$ & $0 . .2$ & Assumed \\
\hline$\delta_{2}$ & $\begin{array}{l}\text { probability of the individuals to be passive } \\
\text { infected from } R\end{array}$ & 0.2 & Assumed \\
\hline$\delta_{1}$ & $\begin{array}{l}\text { probability of the individuals entering I from } \\
\text { E }\end{array}$ & 0.045 & Assumed \\
\hline$r$ & natural recovery rate from $\mathrm{V}$ & 0.14286 per year & $\begin{array}{l}\text { (Stephen et al, } \\
\text { 2014) }\end{array}$ \\
\hline$\lambda$ & force of infection & 0.096 per year & $\begin{array}{l}\text { (Stephen et al, } \\
\text { 2014) }\end{array}$ \\
\hline$\rho$ & natural recovery from I & 0.003 & Assumed \\
\hline
\end{tabular}

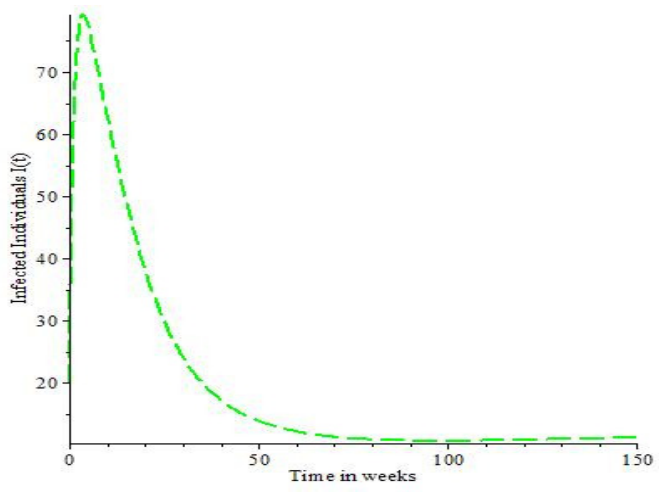

Fig 1: Simulation of Infected population

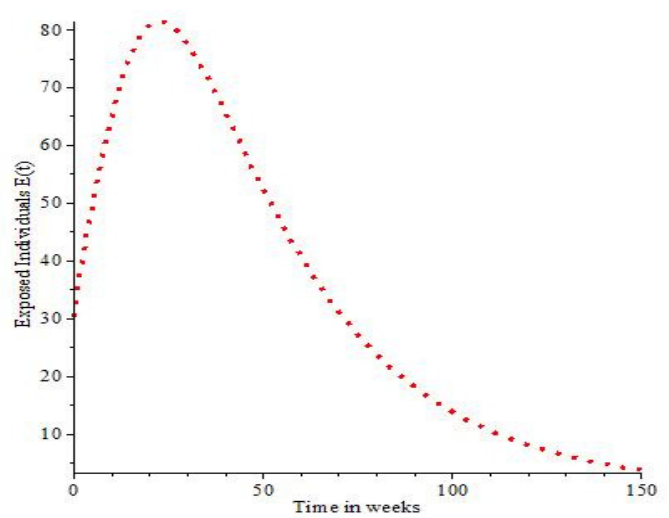

Fig 2: Simulation of Exposed population 


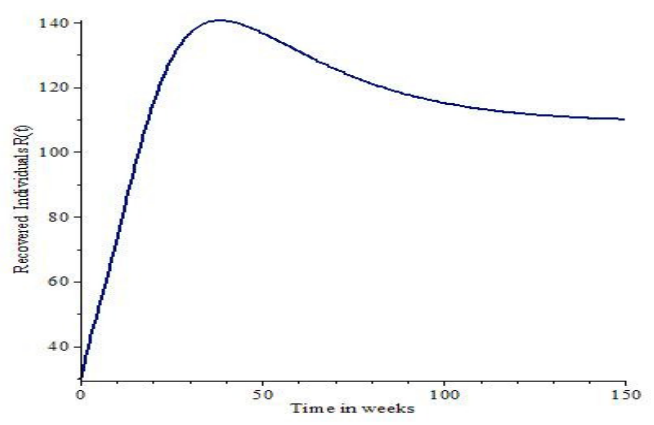

Fig 3: Simulation of Recovered population

Assuming vaccination is effective in protecting susceptible individuals, Figures 1 and 2 show that vaccination can reduce the peak of exposed and infected compartment drastically. Figure 3 shows the dynamics of recovered compartment with vaccination it was observed that the population of the recovered individuals at the very beginning rise rapidly as the rate increases and then fall as time increases. This rapid decline of the infected individuals may be due to early detection of the measles and partly due to those who revert to the Exposed class

Conclusion: In this paper we have developed a mathematical model for the transmission of measles disease by considering recurrent infection and vaccination. The disease free equilibrium and its stability is presented and its relation to the basic reproduction number and vaccination reproduction number is discussed. Numerical examples show that vaccination is able to prevent the disease from spreading. Furthermore, we have shown that $R_{v}>1$ implies that the disease will persist, which implies that $R_{V}=1$ is the threshold between the extinction of the disease and the persistence of the disease.

\section{REFERENCES}

World Health Organization, Department of vaccinces and biologicals, (2005). Measles Technical Working Group: strategies for measles control and elimination. Report of a meeting, Geneva, 1112 May .Geneva, Switzerland

Simons E; Ferrari M; Fricks Kathleen W; Abhijeet A (2012). Assessment of the 2010 global measles mortality reduction http://www.thelancet.com. https://doi.org/10.1016/S0140-6736 (12)60522-4 goal: results from a model of surveillance data (Accessed June 2016).

Grais, R; Ferrari, M; Bjrnstad, O; Grenfell, B; Djibo, A; Fermon, F; Guerin, $\mathrm{P}(2006)$ Estimating transmission intensity for a measles epidemic in Niamey, Niger: lessons for intervention.
Transactions of the Royal Society of Tropical Medicine and Hygiene (2006) (In Press).

Ejima K, Omori R, Aihara K, Nishiura, H (2012) Real-time investigation of measles epidemics with estimate of vaccine efficacy. Int J Biol Sci.; 8(5):620-629.

Bakare, EA; Adekunle, YA; Kadiri, KO (2012) Modelling and Simulation of the Dynamics of the Transmission of Measles. Inter. J. Computer Trends and Technol. 3:174-178

Bolarian, G (2014). On the Dynamical Analysis of a new Model for Measles Infection. Inter. J. Math. Trends Technol. 7:144-155.

Verguet S, (2015). Controlling measles using supplemental immunization activities: A mathematical model to inform optimal policy. Vaccine http://dx.doi.org/10.1016/j.vaccine.2017.11.050

Momoh, AA; Ibrahim, MO; Uwanta, IJ; Manga, SB (2013). Mathematical Model for control of Measles Epidemiology. Inter. J. Pure Appl. Math. 67: 707-718

Comiskey B A (Mod). (1988) A mathematical Model for Measles Epidemics in Ireland. Msc Thesis. National Institute of Higher Education.

Derrick, NR; Grossman, SL (1976). Differential Equation with application. Addision Wesley Publishing Company, Inc. Philippines.

Stephen, E., Dmitry, K; Silas, M (2014). Modeling the Impact of Immunization on the epidemiology of Varicella Zoster Virus. Math. Theo. Model. 4 (8):46-56.

Sarah A. (2012). Global Journal of Science Frontier Research Mathematics and Decision Sciences 12(14):58-66

Anes Tawhir. (2012). Modelling and Control of Measles Transmission in Ghana. Master of Philosophy thesis. Kwame Nkrumah University of Science and Technology.

Fred, MO; Sigey, JK; Okello, JA. Okwyo, JM; Kang' ethe, GJ (2014) Mathematical Modelling on the Control of Measles by Vaccination: Case Study of KISII Country, Kenya. SIJ Transact. Comp. Sci. Engineer. Applicat. (CSEA), 2: 61-69 